M. Sheikh ${ }^{\mathrm{a}}$

M. Osman ${ }^{\mathrm{b}}$

a Department of Radiology, Faculty of Medicine, Kuwait University, and

b Department of Radiology, Mubarak Al-Kabeer Hospital, Kuwait

\title{
Spinal Extradural Hydatid Cyst
}

\section{Key Words}

Intraspinal hydatid cysts

CT scan

MRI investigation

\begin{abstract}
Objective and Importance: Hydatid disease of the bones is uncommon. Spinal hydatid disease presenting with compressive neuropathy is very rare. This case is presented for the rarity of this condition. Clinical Presentation: An adult male patient with previously treated hydatid cyst of the liver presented with symptoms of spinal nerve root compression. MRI of the spine revealed an extradural cyst which was treated with surgical excision. Conclusion: Patients with a known history of hydatidosis should be suspected of spinal hydatid when presenting with symptoms or signs of cord or nerve root compression.
\end{abstract}

\section{Introduction}

Hydatid disease is an infestation by the larval stages of Echinococcus granulosus which can encyst in a number of organs. In man, the liver and lungs are the organs most frequently involved. Bone involvement occurs in $1 \%$ of the cases, with the vertebral column being involved in $50 \%$ of these cases. Extension into the spinal canal results in spinal cord compression [1,2]. We report a rare case of nerve root compression caused by an intraspinal

\begin{tabular}{ll}
\hline KARGER & ( 1998 S. Karger AG, Basel \\
Fax +4161306 1234 & 1011-7571/98/0074-0306\$15.00/0 \\
$\begin{array}{l}\text { E-Mail karger@karger.ch } \\
\text { www.karger.com }\end{array}$ & $\begin{array}{l}\text { Accessible online at: } \\
\text { http://BioMedNet.com/karger }\end{array}$
\end{tabular}

extradural hydatid cyst. To the best of our knowledge, this is the first case reported in this part of the Middle East.

\section{Case Report}

A 40-year-old male patient was admitted to the hospital with numbness in the front part of both his thighs and a feeling of heaviness in his legs while walking. An examination revealed a relative weakness of flexion at both hip joints with an almost complete foot drop of the left foot. Fourteen months earlier, he had
Dr. M. Sheikh

Department of Radiology, Faculty of Medicine, Kuwait University PO Box 24923, 13110 Safat (Kuwait)

Tel. +965 5330473 , Fax +965 5338936

E-Mail mehraj@hsc.kuniv.edu.kw 
Fig. 1. $T_{1}$ - and $T_{2}$-weighted sagittal images of the lumbar spine showing the cyst extradurally at the level of $\mathrm{L}_{2}$ (arrows). The conus is compressed. a $\mathrm{T}_{1}$-weighted image. b $\mathrm{T}_{2}$-weighted image. a

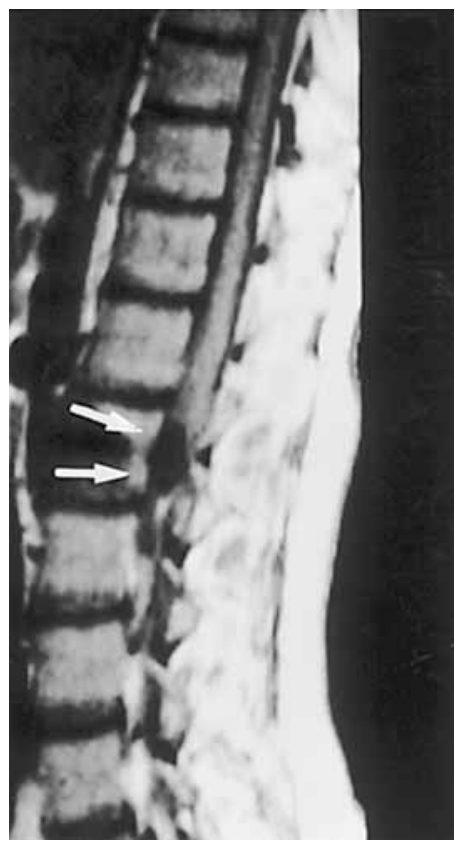

b

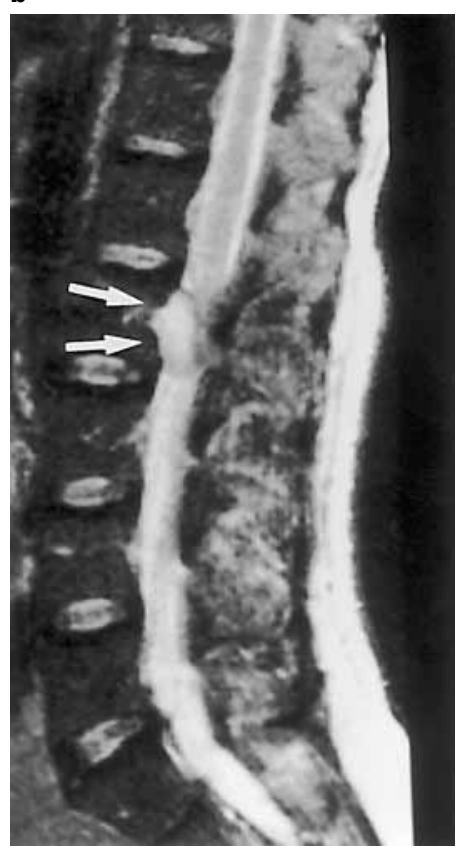

undergone marsupialisation of a hydatid cyst of the right lobe of the liver. The chest radiograph was normal at the time of admission. MRI of the lumbar spine in $\mathrm{T}_{1^{-}}$and $\mathrm{T}_{2}$-weighted scans was performed and showed a small extradural intraspinal cyst at the level of the $\mathrm{L}_{2}$ vertebra, compressing the tip of the conus (fig. 1). There was also a cystic mass noted in the right paravertebral region. Low and intermediate signal changes were noted in the body of the $\mathrm{L}_{2}$ vertebra on $\mathrm{T}_{1}$ - and $\mathrm{T}_{2}$-weighted images, respectively. On the basis of these findings and previous history, hydatidosis was diagnosed and treatment with mebendazole was started and continued for 3 months. A CT scan of the abdomen with intravenous contrast medium was performed for proper evaluation of the paravertebral cyst. $\mathrm{CT}$ confirmed the cyst in the right paraspinal region involving the psoas muscle (fig. 2a). Magnified images of the $\mathrm{L}_{2}$ vertebra in bone windows revealed patchy sclerotic changes in the body of this vertebra (fig. 2b). The patient underwent surgery in Germany for excision of the paraspinal and intraspinal cyst. A follow-up scan after 18 months showed no recurrence (fig. 3) and the patient was symptom free.

\section{Discussion}

Hydatid disease is endemic in some countries, including the Middle East [1]. Although cases of spinal hydatidosis have been reported, a solitary extradural location of the hydatid cyst is extremely rare [2-4]. Primary extradural intraspinal hydatid cysts are considered to arise from an undetected bony focus $[5,6]$.

The development of neurological signs from these cysts indicates extradural compression of the cord, which may result from the cysts involving the vertebrae; or if the cysts arise in the paravertebral area as in our case, there can be an erosion of the vertebrae causing extradural cord or nerve root compression [5-8]. CT and MRI provide different but complementary evidence for the diagnosis of hydatid disease, with CT in particular being helpful in its role to determine bone destruction. MRI, especially $\mathrm{T}_{2}$ scans, clearly 


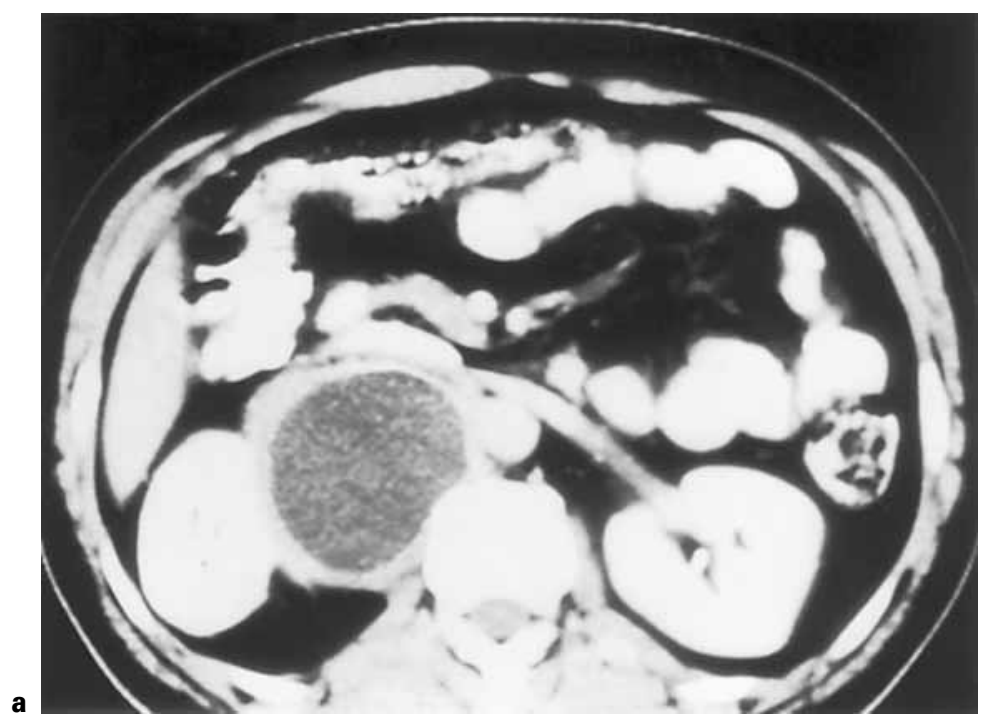

Fig. 2. a CT scan of the abdomen showing the cyst in the right paravertebral location. b Magnified view of the $\mathrm{L}_{2}$ vertebra in the bone window shows irregular sclerotic changes.

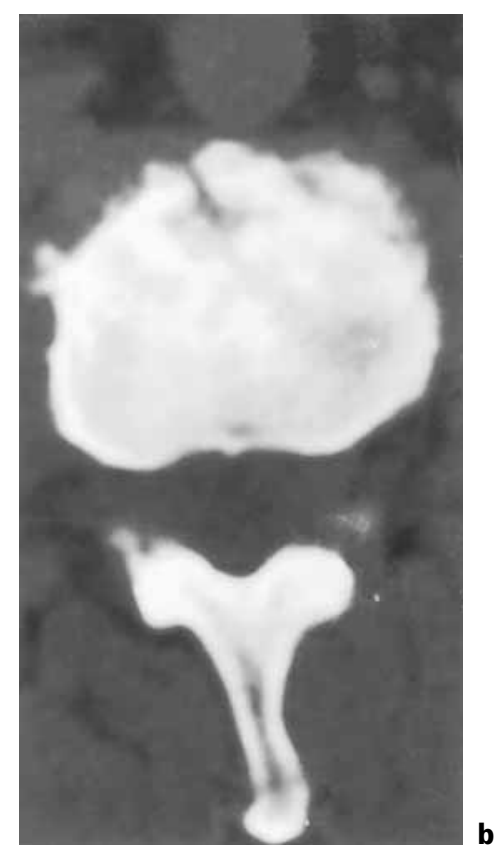

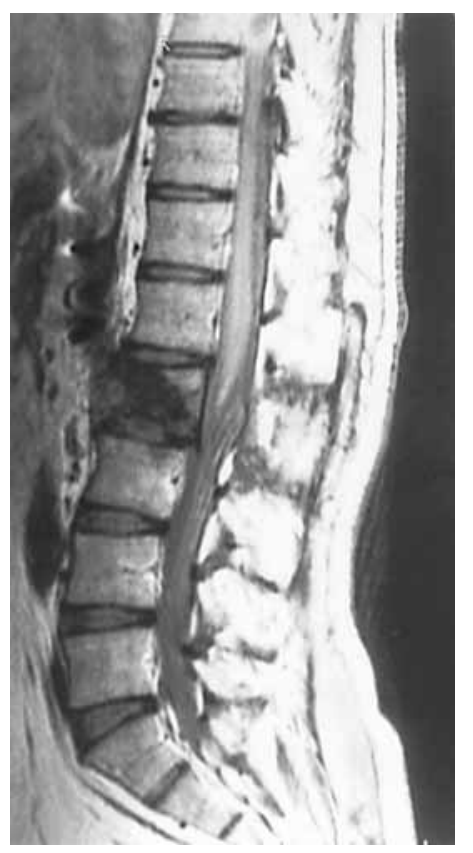

Fig. 3. $T_{1}$-weighted sagittal scan of the lumbar spine showing that the cyst has been removed. reveals the cysts and defines their size, extent and relation to the cord $[5,7,9,10]$. In our case, the large cyst in the right paravertebral area and the bone changes were clearly demonstrated by CT. MRI was superior in demonstrating the intraspinal cyst compressing the cord.

Concerning management, surgical decompression in association with mebendazole is recommended. The progress with this therapy is promising, but it is felt that recurrence is inevitable when vertebrae are affected by the microvascular type of hydatid cysts [5, 7, 9].

\section{Conclusion}

In conclusion therefore, patients with a known history of hydatidosis should be suspected of spinal hydatid disease when presenting with symptoms or signs of cord or nerve root compression. 


\section{References}

1 Matossion RM, Richard MD Symth JD: Hydatidosis: A global problem of increasing importance. WHO Chron 1977;55:499-507.

2 Medjek L, Zenini S, Hamnoon S, Hartani M: Intradural hydatidosis of the thoracic spine: Apropos of a case. Ann Radiol (Paris) 1991;34: 251-255.

3 Akhan O, Dincer A, Saatci I, Gulekon N, Besim A: Spinal intradural hydatid cyst in a child. Br J Radiol 1991;64:465-466.
4 Iplikcioglu AC, Kokes F, Bayar A, Doganay S, Buharali S: Spinal invasion of pulmonary hydatidosis: Computed tomographic demonstration. Neurosurgery 1991;29:467468.

5 Payport M, Wisoff HS, Zalman H: Vertebral echinococcosis. J Neurosurg 1964;21:647-660.

6 Pamir MN, Akalan N, Ozgen T, Erbengi A: Spinal hydatid cyst. Surg Neurol 1984;21:53-57.

7 Charles RW, Govender S, Naidoo KS: Echinococcal infection of the spine with neural involvement. Spine 1988;13:47-49.
8 Kars HZ, Hekimoglu B, Cepoglu C: Spinal epidural hydatid cyst. Radiological and ultrasonographical workup of a case. Eur J Radiol 1990; 19:212-214.

9 Wan MA, Taheri SA, Babu ML, Ahangar GA, Wan H: Primary extradural hydatid cyst. Neurosurgery 1989;24:631-632.

10 Pau A, Simonetti G, Tortari-Donat P, Turtas S, Viale GL: Computed tomography and magnetic resonance imaging in spinal hydatidosis. Surg Neurol 1987;27:365-369. 